\title{
Analytic and numerical solutions for systems of fractional Schrödinger equation
}

\author{
Rabha W Ibrahim ${ }^{1 *}$ and Hamid A Jalab²
}

\section{"Correspondence:}

rabhaibrahim@yahoo.com

${ }^{1}$ Institute of Mathematical Sciences, University of Malaya, Kuala Lumpur, 50603, Malaysia

Full list of author information is

available at the end of the article

\begin{abstract}
In this work, we address the existence and uniqueness of a fractional system involving nonlinear Schrödinger equations. This system of fractional partial differential equations arises in quantum mechanics. It describes how the quantum state of some physical system changes with time. We show that the system under consideration admits a global solution in appropriate functional spaces. The solution is shown to be unique. The technique is established as an analytic technique of the fixed point theorem. We suppose that all the functions are analytic in the open unit disk. The fractional differential operator is considered from the point of view of the Riemann-Liouville differential operator. Moreover, a numerical scheme for this solution is calculated.
\end{abstract}

\section{Introduction}

It is well known that the Schrödinger equation comes in two kinds. The first type is the one in which time explicitly appears, and so it describes how the wave function of a particle will promote in time. In general, the wave function performs like a wave, and thus the equation is often called the time dependent Schrödinger wave equation. The second type is the equation in which the time has been extracted and thus is referred to as the time independent Schrödinger equation.

In recent years, the fractional calculus is considered as the best tool for the generalizations of fractional differential equations [1-5]. In 2000, Laskin imposed the fractional Schrödinger equation, in which the normal Schrödinger equation is generalized in analogy with fractional diffusion. Laskin professed to exactly solve this equation in the case of the one-dimensional infinite square well [6]. In 2006, Guo and Xu claimed to have introduced solutions again for the infinite one-dimensional square well (agreeing with the Laskin original solution), and for one-dimensional scattering off a barrier potential [7]. Again in 2007, Laskin utilized a different method of analysis to pose solutions for the linear, delta function, and Coulomb potentials in one dimension [8]. Recently, different studies have been published [9-11].

Numerical methods, for finding solutions of the time-fractional nonlinear Schrödinger equation in one and two dimensions, have appeared in quantum mechanics. Recently, Ford et al. prepared a numerical method for the fractional Schrödinger type equation of spatial dimension two [12]. The existence and uniqueness of fractional Schrödinger equations are studied by many authors (see [13]). 
In this paper, we establish the existence and uniqueness of fractional system involving nonlinear Schrödinger equations. This system of time-fractional partial differential equations arises in quantum mechanics. It describes how the quantum state of some physical system changes with time. We show that the system under consideration admits a global solution in appropriate functional spaces. The solution is shown to be unique. The tool based on an analytic technique from the fixed point theory and the fractional calculus is taken in the sense of the Riemann-Liouville differential operator. We suppose that all the functions are analytic in the open unit disk.

\section{Generalized calculus}

A fractional of arbitrary order originates from the Riemann-Liouville definition of a generalized integral with order $\alpha$ as follows [3, 4]:

$$
{ }_{a} I_{t}^{\alpha} f(t)=\int_{a}^{t} \frac{(t-\tau)^{\alpha-1}}{\Gamma(\alpha)} f(\tau) d \tau
$$

The derivative operator of arbitrary order for the function $f$ of order $\alpha>0$ is read by

$$
{ }_{a} D_{t}^{\alpha} f(t)=(d / d t) \int_{a}^{t} \frac{(t-\tau)^{-\alpha}}{\Gamma(1-\alpha)} f(\tau) d \tau
$$

When $a=0$, we shall denote ${ }_{0} D_{t}^{\alpha} f(t):=D_{t}^{\alpha} f(t)$ and ${ }_{0} I_{t}^{\alpha} f(t):=I_{t}^{\alpha} f(t)$ in the follow-up.

Newly, Alsaedi et al. [14] derived an inequality for fractional derivatives as follows.

Lemma 2.1 Let one of the following assumptions be satisfied:

- $u \in C([0, T])$ and $v \in C^{\beta}([0, T]), \alpha<\beta \leq 1$,

- $v \in C([0, T])$ and $u \in C^{\beta}([0, T]), \alpha<\beta \leq 1$,

- $u \in C^{\beta}([0, T])$ and $v \in C^{\delta}([0, T]), \alpha<\beta \leq \beta+\delta, \beta, \delta \in(0,1)$,

where $C^{\gamma}([0, T])=\left\{u:[0, T] \rightarrow \mathbb{R} /|u(t)-u(t-h)|=O\left(h^{\gamma}\right)\right.$ uniformly for $\left.0<t-h<t \leq T\right\}$.

Then we have

$$
\begin{aligned}
D_{t}^{\alpha}(u v)(t)= & u(t) D_{t}^{\alpha} v(t)+v(t) D_{t}^{\alpha} u(t) \\
& -\frac{\alpha}{\Gamma(1-\alpha)} \int_{0}^{t} \frac{(u(s)-u(t))(v(s)-v(t))}{(t-s)^{\alpha+1}} d s \\
& -\frac{u(t) v(t)}{\Gamma(1-\alpha) t^{-\alpha}}
\end{aligned}
$$

point-wise.

Remark 2.1 In virtue of Lemma 2.1, if $v$ and $u$ have the same sign and are both increasing or both decreasing, then

$$
D_{t}^{\alpha}(u v)(t) \leq u(t) D_{t}^{\alpha} v(t)+v(t) D_{t}^{\alpha} u(t)
$$

and for $u=v$, we get

$$
D_{t}^{\alpha}\left(u^{2}\right)(t) \leq 2 u(t) D_{t}^{\alpha} u(t)
$$




\section{Fractional system}

Consider the two dimensional fractional system (the nonlinear case can be found in [15])

$$
\begin{aligned}
& i \partial_{t}^{\alpha} \phi_{1}=-\frac{1}{2} \triangle \phi_{1}+\frac{\beta^{2}}{2}|z|^{2} \phi_{1}+\gamma_{11}\left|\phi_{1}\right|^{\mu} \phi_{1}+\gamma_{12}\left|\phi_{2}\right|^{2} \phi_{1}+\lambda \phi_{2}, \\
& i \partial_{t}^{\alpha} \phi_{2}=-\frac{1}{2} \triangle \phi_{2}+\frac{\beta^{2}}{2}|z|^{2} \phi_{2}+\gamma_{12}\left|\phi_{1}\right|^{2} \phi_{2}+\gamma_{22}\left|\phi_{2}\right|^{\mu} \phi_{2}+\lambda \phi_{1},
\end{aligned}
$$

where $z \in U:=\{z \in \mathbb{C}:|z|<1\}, t>0$ and $\partial_{t}^{\alpha}$ is the Riemann-Liouville differential operator which is defined by $\partial_{t}^{\alpha} \phi(t)=D_{t}^{\alpha} \phi(t)$, subject to the initial conditions

$$
\left(\phi_{1}(0, \cdot)=\phi_{1}^{0}, \phi_{2}(0, \cdot)=\phi_{2}^{0} \in U\right)
$$

The parameter set can be listed as

- $i$ is the imaginary unit;

- $\alpha \in(0,1]$ is the fractional order;

- $\triangle$ is the Laplacian;

- $\beta>0$ is referred as the magnetic trapping strength;

- $\mu>0$ is the mean-field exponent;

- $\lambda \in \mathbb{R}$ is the external driven field constant;

- $\gamma_{i j}$ is for the intra specific scattering lengths;

- $\phi_{1}, \phi_{2}$ are wave functions of a quantum system.

The system can be written in the compact form

$$
i \partial_{t}^{\alpha} \Phi=A \Phi+F(\Phi)+B \Phi, \quad \Phi(\cdot, 0)=\Phi^{0},
$$

where

$$
A=\left(\begin{array}{cc}
-\frac{1}{2} \Delta+\frac{1}{2} \beta^{2}|z|^{2} & 0 \\
0 & -\frac{1}{2} \Delta+\frac{1}{2} \beta^{2}|z|^{2}
\end{array}\right), \quad B=\left(\begin{array}{ll}
0 & \lambda \\
\lambda & 0
\end{array}\right),
$$

and

$$
F(\Phi)=\left(\begin{array}{c}
\gamma_{11}\left|\phi_{1}\right|^{\mu} \phi_{1}+\gamma_{12}\left|\phi_{2}\right|^{2} \phi_{1} \\
\gamma_{12}\left|\phi_{1}\right|^{2} \phi_{2}+\gamma_{22}\left|\phi_{2}\right|^{\mu} \phi_{2}
\end{array}\right)
$$

It is clear that $F(\Phi)$ and $B \Phi$ are locally Lipschitz continuous in the unit disk.

\section{Existence solution}

In this section, we deduce the existence and uniqueness for the system (3.1a)-(3.1b) with the initial condition $\phi_{1}(0, \cdot)=\phi_{1}^{0}, \phi_{2}(0, \cdot)=\phi_{2}^{0}$.

Theorem 4.1 Consider $\left(\phi_{1}^{0}, \phi_{2}^{0}\right) \in H^{1}(U)^{2}$ and $\left|\gamma_{j j}\right| \leq\left|\gamma_{12}\right|, j=1$, 2. If $\frac{\sigma T^{\alpha}}{\Gamma(\alpha+1)}<1$, for a positive number $\sigma$, then there exists a unique global solution $\left(\phi_{1}, \phi_{2}\right)$ for the system (3.1a)-(3.1b) such that

$$
\left(\phi_{1}, \phi_{2}\right) \in L^{\infty}\left([0, T], H^{1}\right) \cap L^{2}\left([0, T], H^{2}\right) \times L^{\infty}\left([0, T], H^{1}\right) \cap L^{2}\left([0, T], H^{2}\right) .
$$


Moreover,

$$
\left(\partial_{t}^{\alpha} \phi_{1}, \partial_{t}^{\alpha} \phi_{2}\right) \in\left(L^{2}\left([0, T] ; L^{2}\right) \times C\left([0, T] ; L^{2}\right) \times L^{2}\left([0, T] ; L^{2}\right) \times C\left([0, T] ; L^{2}\right)\right) .
$$

Proof The proof takes place in six steps. The first five steps concern prior estimates. Step 6 addresses uniqueness.

Step 1. Formal first energy estimates. Multiplying (3.1a) by $\phi_{1}$, integrating over the unit disk $U$, and applying Remark 2.1, we obtain the first estimate

$$
\begin{aligned}
\frac{1}{2} \partial_{t}^{\alpha}\left\|\phi_{1}(t, \cdot)\right\|_{L^{2}}^{2}+\frac{1}{2}\left\|\frac{\partial \phi_{1}}{\partial z}(t, \cdot)\right\|_{L^{2}}^{2} \leq & \frac{\beta^{2}}{2} \int_{U}|z|^{2}\left|\phi_{1}\right|^{2}+\frac{\left|\gamma_{11}\right|}{\mu+2}\left\|\phi_{1}(t, \cdot)\right\|_{L^{\mu+2}}^{\mu+2} \\
& +\left|\gamma_{12}\right| \int_{U}\left|\phi_{2}(t, \cdot)\right|^{2}\left|\phi_{1}(t, \cdot)\right|^{2} d z+\lambda \int_{U} \phi_{1} \phi_{2} \\
\leq & \frac{\beta^{2}}{2}\left\|\phi_{1}\right\|_{L^{2}}^{2}+\frac{\left|\gamma_{11}\right|}{\mu+2}\left\|\phi_{1}(t, \cdot)\right\|_{L^{\mu+2}}^{\mu+2} \\
& +\left|\gamma_{12}\right| \int_{U}\left|\phi_{2}(t, \cdot)\right|^{2}\left|\phi_{1}(t, \cdot)\right|^{2}+\lambda \int_{U} \phi_{1} \phi_{2} .
\end{aligned}
$$

Similarly, we multiply (3.1b) by $\phi_{2}$ and integrate over $U$ to get

$$
\begin{aligned}
\frac{1}{2} \partial_{t}^{\alpha}\left\|\phi_{2}(t, \cdot)\right\|_{L^{2}}^{2}+\frac{1}{2}\left\|\frac{\partial \phi_{2}}{\partial z}(t, \cdot)\right\|_{L^{2}}^{2} \leq & \frac{\beta^{2}}{2} \int_{U}|z|^{2}\left|\phi_{2}\right|^{2}+\frac{\left|\gamma_{22}\right|}{\mu+2}\left\|\phi_{2}(t, \cdot)\right\|_{L^{\mu+2}}^{\mu+2} \\
& +\left|\gamma_{12}\right| \int_{U}\left|\phi_{1}(t, \cdot)\right|^{2}\left|\phi_{2}(t, \cdot)\right|^{2} d z+\lambda \int_{U} \phi_{1} \phi_{2} \\
\leq & \frac{\beta^{2}}{2}\left\|\phi_{2}\right\|_{L^{2}}^{2}+\frac{\left|\gamma_{22}\right|}{\mu+2}\left\|\phi_{2}(t, \cdot)\right\|_{L^{\mu+2}}^{\mu+2} \\
& +\left|\gamma_{12}\right| \int_{U}\left|\phi_{1}(t, \cdot)\right|^{2}\left|\phi_{2}(t, \cdot)\right|^{2}+\lambda \int_{U} \phi_{1} \phi_{2} .
\end{aligned}
$$

Combining (4.3) and (4.4), we have

$$
\begin{aligned}
\frac{1}{2}\left(\left\|\phi_{1}\right\|_{L^{2}}^{2}+\left\|\phi_{2}\right\|_{L^{2}}^{2}\right)+\frac{1}{2}\left(\left\|\frac{\partial \phi_{1}}{\partial z}\right\|_{L^{2}}^{2}+\left\|\frac{\partial \phi_{2}}{\partial z}\right\|_{L^{2}}^{2}\right) \\
\leq \frac{\beta^{2}}{2}\left(\left\|\phi_{1}\right\|_{L^{2}}^{2}+\left\|\phi_{2}\right\|_{L^{2}}^{2}\right)+\frac{1}{\mu+2}\left(\left|\gamma_{11}\right|\left\|\phi_{1}\right\|_{L^{\mu+2}}^{\mu+2}+\left|\gamma_{22}\right|\left\|\phi_{2}(t, \cdot)\right\|_{L^{\mu+2}}^{\mu+2}\right) \\
\quad+2\left|\gamma_{12}\right| \int_{U}\left|\phi_{1}(t, \cdot)\right|^{2}\left|\phi_{2}(t, \cdot)\right|^{2}+2 \lambda \int_{U} \phi_{1} \phi_{2} \\
\leq \frac{\beta^{2}}{2}\left(\left\|\phi_{1}(t, \cdot)\right\|_{L^{2}}^{2}+\left\|\phi_{2}(t, \cdot)\right\|_{L^{2}}^{2}\right)+\frac{1}{\mu+2}\left(\left|\gamma_{11}\right|\left\|\phi_{1}(t, \cdot)\right\|_{L^{\mu+2}}^{\mu+2}+\left|\gamma_{22}\right|\left\|\phi_{2}(t, \cdot)\right\|_{L^{\mu+2}}^{\mu+2}\right) \\
\quad+2\left|\gamma_{12}\right|\left\|\phi_{1}(t, \cdot)\right\|_{L^{4}}^{2}\left\|\phi_{2}(t, \cdot)\right\|_{L^{4}}^{2}+2|\lambda|\left\|\phi_{1}(t, \cdot)\right\|_{L^{2}}\left\|\phi_{2}(t, \cdot)\right\|_{L^{2}} \cdot
\end{aligned}
$$

Employing the Gagliardo-Nirenberg inequality and utilizing the inequality

$$
\|\varphi(t, \cdot)\|_{L^{2}} \leq \frac{\|\varphi(t, \cdot)\|_{L^{2}}^{2}+1}{2}
$$


yield

$$
\begin{aligned}
& \frac{1}{2}\left(\left\|\phi_{1}(t, \cdot)\right\|_{L^{2}}^{2}+\left\|\phi_{2}(t, \cdot)\right\|_{L^{2}}^{2}\right)+\frac{1}{2}\left(\left\|\nabla \phi_{1}(t, \cdot)\right\|_{L^{2}}^{2}+\left\|\nabla \phi_{2}(t, \cdot)\right\|_{L^{2}}^{2}\right) \\
& \leq \frac{\beta^{2}}{2}\left(\left\|\phi_{1}\right\|_{L^{2}}^{2}+\left\|\phi_{2}\right\|_{L^{2}}^{2}\right) \\
& \quad+\frac{K_{\mu}}{\mu+2}\left(\left|\gamma_{11}\right|\left\|\nabla \phi_{1}(t, \cdot)\right\|_{L^{2}}^{\mu}\left\|\phi_{1}(t, \cdot)\right\|_{L^{2}}^{2}+\left|\gamma_{22}\right|\left\|\nabla \phi_{2}(t, \cdot)\right\|_{L^{2}}^{\mu}\left\|\phi_{2}(t, \cdot)\right\|_{L^{2}}^{2}\right) \\
& \quad+2 K\left|\gamma_{12}\right|\left\|\nabla \phi_{1}(t, \cdot)\right\|_{L^{2}}\left\|\phi_{1}(t, \cdot)\right\|_{L^{2}}\left\|\nabla \phi_{2}(t, \cdot)\right\|_{L^{2}}\left\|\phi_{2}(t, \cdot)\right\|_{L^{2}} \\
& \quad+2|\lambda|\left\|\phi_{1}(t, \cdot)\right\|_{L^{2}}\left\|\phi_{2}(t, \cdot)\right\|_{L^{2}} \\
& \leq \frac{\beta^{2}}{2}\left(\left\|\phi_{1}(t, \cdot)\right\|_{L^{2}}^{2}+\left\|\phi_{2}(t, \cdot)\right\|_{L^{2}}^{2}\right) \\
&+\frac{K_{\mu}}{\mu+2}\left(\left|\gamma_{11}\right|\left\|\nabla \phi_{1}(t, \cdot)\right\|_{L^{2}}^{\mu}\left\|\phi_{1}(t, \cdot)\right\|_{L^{2}}^{2}+\left|\gamma_{22}\right|\left\|\nabla \phi_{2}(t, \cdot)\right\|_{L^{2}}^{\mu}\left\|\phi_{2}(t, \cdot)\right\|_{L^{2}}^{2}\right) \\
&+K\left|\gamma_{12}\right|\left(\left\|\nabla \phi_{1}(t, \cdot)\right\|_{L^{2}}^{2}\left\|\phi_{1}(t, \cdot)\right\|_{L^{2}}^{2}+\left\|\nabla \phi_{2}(t, \cdot)\right\|_{L^{2}}^{2}\left\|\phi_{2}(t, \cdot)\right\|_{L^{2}}^{2}\right) \\
&+|\lambda|\left(\left\|\phi_{1}\right\|_{L^{2}}^{2}+\left\|\phi_{2}\right\|_{L^{2}}^{2}\right)+|\lambda|,
\end{aligned}
$$

where $K_{\mu}>0$ and $K>0$. Now let $\left\|\nabla \phi_{1}(t, \cdot)\right\|_{L^{2}}^{m_{1}}:=\max \left\{\left\|\nabla \phi_{1}(t, \cdot)\right\|_{L^{2}}^{2},\left\|\nabla \phi_{1}(t, \cdot)\right\|_{L^{2}}^{\mu}\right\}$ and $\left\|\nabla \phi_{2}(t, \cdot)\right\|_{L^{2}}^{m_{2}}:=\max \left\{\left\|\nabla \phi_{2}(t, \cdot)\right\|_{L^{2}}^{2},\left\|\nabla \phi_{2}(t, \cdot)\right\|_{L^{2}}^{\mu}\right\}$, we conclude that

$$
\begin{aligned}
& \frac{1}{2} \partial_{t}^{\alpha}\left(\left\|\phi_{1}(t, \cdot)\right\|_{L^{2}}^{2}+\left\|\phi_{2}(t, \cdot)\right\|_{L^{2}}^{2}\right)+\frac{1}{2}\left(\left\|\nabla \phi_{1}(t, \cdot)\right\|_{L^{2}}^{2}+\left\|\nabla \phi_{2}(t, \cdot)\right\|_{L^{2}}^{2}\right) \\
& \leq\left(|\lambda|+\frac{\beta^{2}}{2}\right)\left(\left\|\phi_{1}\right\|_{L^{2}}^{2}+\left\|\phi_{2}\right\|_{L^{2}}^{2}\right) \\
& \quad+\left(\frac{K_{\mu}}{\mu+2}+K\right)\left|\gamma_{12}\right|\left(\left\|\nabla \phi_{1}(t, \cdot)\right\|_{L^{2}}^{m_{1}}\left\|\phi_{1}(t, \cdot)\right\|_{L^{2}}^{2}+\left\|\nabla \phi_{2}(t, \cdot)\right\|_{L^{2}}^{m_{2}}\left\|\phi_{2}(t, \cdot)\right\|_{L^{2}}^{2}\right)+|\lambda| .
\end{aligned}
$$

By applying the generalized Gronwall lemma to the last assertion, we find that

$$
\begin{aligned}
& \sup _{t \in[0, T]}\left(\left\|\phi_{1}(t, \cdot)\right\|_{L^{2}}^{2}+\left\|\phi_{2}(t, \cdot)\right\|_{L^{2}}^{2}\right)+\int_{0}^{T}\left(\left\|\phi_{1}(t, \cdot)\right\|_{H^{1}}^{2}+\left\|\phi_{2}(t, \cdot)\right\|_{H^{1}}^{2}\right) d t \\
& \leq \int_{0}^{T}\left(\left\|\phi_{1}(t, \cdot)\right\|_{L^{2}}^{2}+\left\|\phi_{2}(t, \cdot)\right\|_{L^{2}}^{2}\right) d t \\
& \quad+\int_{0}^{T}\left(\left\|\nabla \phi_{1}(t, \cdot)\right\|_{L^{2}}^{m_{1}}\left\|\phi_{1}(t, \cdot)\right\|_{L^{2}}^{2}+\left\|\nabla \phi_{2}(t, \cdot)\right\|_{L^{2}}^{m_{2}}\left\|\phi_{2}(t, \cdot)\right\|_{L^{2}}^{2}\right) d t+C_{0, T}
\end{aligned}
$$

where $C_{0, T}$ is a constant depending on $\alpha, \beta, \mu, K_{\mu}, K,\left|\gamma_{12}\right|, T,|\lambda|, \phi_{1}^{0}$, and $\phi_{2}^{0}$.

Step 2. $L^{\infty}$-bounds. In this step, we proceed to compute the $L^{\infty}$-bounds on $\phi_{1}$ and $\phi_{2}$. Multiplying (3.1a) by $\triangle \phi_{1}=\frac{\partial^{2} \phi_{1}}{\partial z^{2}}$ and integrating over $U$ yield

$$
\begin{aligned}
& \frac{1}{2} \partial_{t}^{\alpha}\left(\left\|\frac{\partial}{\partial z} \phi_{1}(t, \cdot)\right\|_{L^{2}}^{2}\right)+\frac{1}{2}\left(\left\|\frac{\partial^{2} \phi_{1}}{\partial z^{2}}(t, \cdot)\right\|_{L^{2}}^{2}\right) \\
& \quad \leq \frac{\beta^{2}}{2} \int_{U}\left|\phi_{1} \frac{\partial^{2} \phi_{1}}{\partial z^{2}}\right|(t, \cdot)+\left|\gamma_{11}\right| \int_{U}\left|\phi_{1}\right|^{\mu}\left|\phi_{1} \frac{\partial^{2} \phi_{1}}{\partial z^{2}}\right|(t, \cdot)
\end{aligned}
$$




$$
\begin{aligned}
& +\left|\gamma_{12}\right| \int_{U}\left|\phi_{2}\right|^{2}\left|\phi_{1} \frac{\partial^{2} \phi_{1}}{\partial z^{2}}\right|(t, \cdot)+|\lambda| \int_{U}\left|\phi_{2} \frac{\partial^{2} \phi_{1}}{\partial z^{2}}\right|(t, \cdot) \\
\leq & \kappa\left(\int_{U}\left|\phi_{1} \frac{\partial^{2} \phi_{1}}{\partial z^{2}}\right|(t, \cdot)+\int_{U}\left|\phi_{1}\right|^{\mu}\left|\phi_{1} \frac{\partial^{2} \phi_{1}}{\partial z^{2}}\right|(t, \cdot)+\int_{U}\left|\phi_{2}\right|^{2}\left|\phi_{1} \frac{\partial^{2} \phi_{1}}{\partial z^{2}}\right|(t, \cdot)\right. \\
& \left.+\int_{U}\left|\phi_{2} \frac{\partial^{2} \phi_{1}}{\partial z^{2}}\right|(t, \cdot)\right),
\end{aligned}
$$

where $\kappa$ is a positive constant depending on $\beta, \lambda, \gamma_{11}$, and $\gamma_{12}$. In view of the Young and the Cauchy-Schwarz inequalities, we deduce that

$$
\begin{aligned}
\partial_{t}^{\alpha} \| & \frac{\partial}{\partial z} \phi_{1}(t, \cdot)\left\|_{L^{2}}^{2}+\right\| \frac{\partial^{2} \phi_{1}}{\partial z^{2}}(t, \cdot) \|_{L^{2}}^{2} \\
& \leq \widetilde{\kappa}\left(\left\|\phi_{1}\right\|_{L^{2}}^{2}+\left\|\phi_{1}\right\|_{L^{\mu+2}}^{\mu+2}+\left\|\phi_{2}\right\|_{L^{3}}^{2}\left\|\phi_{1}\right\|_{L^{3}}+\left\|\phi_{2}\right\|_{L^{2}}^{2}\right),
\end{aligned}
$$

where $\widetilde{\kappa}$ is a positive constant concerning $\alpha, \beta, \mu, \lambda, \gamma_{11}$, and $\gamma_{12}$. Again by employing the Gagliardo-Nirenberg inequality

$$
\partial_{t}^{\alpha}\left\|\frac{\partial}{\partial z} \phi_{1}(t, \cdot)\right\|_{L^{2}}^{2}+\left\|\frac{\partial^{2} \phi_{1}}{\partial z^{2}}(t, \cdot)\right\|_{L^{2}}^{2} \leq \widehat{\kappa}\left(\left\|\phi_{1}\right\|_{L^{2}}^{2}+\left\|\phi_{2}\right\|_{L^{2}}^{2}\right)
$$

where $\widehat{\kappa}$ is a positive constant depending on $\alpha, \beta, \mu, \lambda, \gamma_{11}$, and $\gamma_{12}$. By the regularity assumptions on the initial conditions that $\left.\frac{\partial}{\partial z} \phi_{1}\right|_{t=0} \in L^{2}(U)$, we therefore have

$$
\phi_{1} \in L^{\infty}\left([0, T], H^{1}\right) \cap L^{2}\left([0, T], H^{2}\right) .
$$

Similarly, multiplying (3.1b) by $\triangle \phi_{2}=\frac{\partial^{2} \phi_{2}}{\partial z^{2}}$ and integrating over $U$ leave us with

$$
\phi_{2} \in L^{\infty}\left([0, T], H^{1}\right) \cap L^{2}\left([0, T], H^{2}\right) .
$$

Step 3. Second estimate: converge solution. Consider the system

$$
\begin{aligned}
& i \partial_{t}^{\alpha} \phi_{1 n}=-\frac{1}{2} \triangle \phi_{1 n}+\frac{\beta^{2}}{2}|z|^{2} \phi_{1 n}+\gamma_{11}\left|\phi_{1 n}\right|^{\mu} \phi_{1 n}+\gamma_{12}\left|\phi_{2 n}\right|^{2} \phi_{1 n}+\lambda \phi_{2 n}, \\
& i \partial_{t}^{\alpha} \phi_{2 n}=-\frac{1}{2} \triangle \phi_{2 n}+\frac{\beta^{2}}{2}|z|^{2} \phi_{2 n}+\gamma_{12}\left|\phi_{1 n}\right|^{2} \phi_{2 n}+\gamma_{22}\left|\phi_{2 n}\right|^{\mu} \phi_{2 n}+\lambda \phi_{1 n},
\end{aligned}
$$

subject to the initial conditions

$$
\left(\phi_{1 n}(0, \cdot)=\phi_{1 n}^{0}, \phi_{2 n}(0, \cdot)=\phi_{2 n}^{0} \in U\right)
$$

In a similar manner to Step 1 , we can see that the approximate solution $\left(\phi_{1 n}, \phi_{2 n}\right)$ is bounded in $L^{2} \times L^{2}$. Multiplying (4.5a) by $\Delta \phi_{1 n}=\frac{\partial^{2} \phi_{1 n}}{\partial z^{2}}$ and integrating over $U$

$$
\begin{aligned}
& \frac{1}{2} \partial_{t}^{\alpha}\left(\left\|\frac{\partial}{\partial z} \phi_{1 n}(t, \cdot)\right\|_{L^{2}}^{2}\right) \\
& \quad \leq \frac{1}{2}\left(\left\|\frac{\partial^{2} \phi_{1 n}}{\partial z^{2}}(t, \cdot)\right\|_{L^{2}}^{2}\right)+\frac{\beta^{2}}{2} \int_{U}\left|\phi_{1 n} \frac{\partial^{2} \phi_{1 n}}{\partial z^{2}}\right|(t, \cdot)+\left|\gamma_{11}\right| \int_{U}\left|\phi_{1 n}\right|^{\mu}\left|\phi_{1 n} \frac{\partial^{2 n} \phi_{1 n}}{\partial z^{2}}\right|(t, \cdot)
\end{aligned}
$$




$$
\begin{aligned}
& +\left|\gamma_{12}\right| \int_{U}\left|\phi_{2 n}\right|^{2}\left|\phi_{1 n} \frac{\partial^{2} \phi_{1 n}}{\partial z^{2}}\right|(t, \cdot)+|\lambda| \int_{U}\left|\phi_{2 n} \frac{\partial^{2} \phi_{1 n}}{\partial z^{2}}\right|(t, \cdot) \\
\leq & \bar{\sigma}\left(\int_{U}\left|\phi_{1 n} \frac{\partial^{2 n} \phi_{1 n}}{\partial z^{2}}\right|(t, \cdot)+\int_{U}\left|\phi_{1 n}\right|^{\mu}\left|\phi_{1 n} \frac{\partial^{2} \phi_{1 n}}{\partial z^{2}}\right|(t, \cdot)+\int_{U}\left|\phi_{2 n}\right|^{2}\left|\phi_{1 n} \frac{\partial^{2} \phi_{1 n}}{\partial z^{2}}\right|(t, \cdot)\right. \\
& \left.+\int_{U}\left|\phi_{2 n} \frac{\partial^{2} \phi_{1 n}}{\partial z^{2}}\right|(t, \cdot)\right),
\end{aligned}
$$

where $\bar{\sigma}$ is a positive constant relating on $\beta, \lambda, \gamma_{11}$, and $\gamma_{12}$. By applying the Young and the Cauchy-Schwarz inequalities and the Gagliardo-Nirenberg inequality, respectively, we propose that

$$
\partial_{t}^{\alpha}\left\|\frac{\partial}{\partial z} \phi_{1 n}(t, \cdot)\right\|_{L^{2}}^{2} \leq \widehat{\sigma}\left(\left\|\phi_{1 n}\right\|_{L^{2}}^{2}+\left\|\phi_{2 n}\right\|_{L^{2}}^{2}+\left\|\frac{\partial^{2} \phi_{1 n}}{\partial z^{2}}(t, \cdot)\right\|_{L^{2}}^{2}\right),
$$

where $\widehat{\sigma}$ is a positive constant depending on $\alpha, \beta, \mu, \lambda, \gamma_{11}$, and $\gamma_{12}$. Similarly for $\phi_{2 n}$ we impose

$$
\partial_{t}^{\alpha}\left\|\frac{\partial}{\partial z} \phi_{2 n}(t, \cdot)\right\|_{L^{2}}^{2} \leq \widetilde{\sigma}\left(\left\|\phi_{1 n}\right\|_{L^{2}}^{2}+\left\|\phi_{2 n}\right\|_{L^{2}}^{2}+\left\|\frac{\partial^{2} \phi_{2 n}}{\partial z^{2}}(t, \cdot)\right\|_{L^{2}}^{2}\right) .
$$

Combining (4.6) and (4.7), then we have

$$
\begin{aligned}
& \partial_{t}^{\alpha}\left(\left\|\frac{\partial}{\partial z} \phi_{1 n}(t, \cdot)\right\|_{L^{2}}^{2}+\left\|\frac{\partial}{\partial z} \phi_{2 n}(t, \cdot)\right\|_{L^{2}}^{2}\right) \\
& \quad \leq \sigma\left(\left\|\phi_{1 n}\right\|_{L^{2}}^{2}+\left\|\phi_{2 n}\right\|_{L^{2}}^{2}+\left\|\frac{\partial^{2} \phi_{1 n}}{\partial z^{2}}(t, \cdot)\right\|_{L^{2}}^{2}+\left\|\frac{\partial^{2} \phi_{2 n}}{\partial z^{2}}(t, \cdot)\right\|_{L^{2}}^{2}\right),
\end{aligned}
$$

where $\sigma:=\widehat{\sigma}+\widetilde{\sigma}$.

Step 4. Third estimate. In this step we argue for an estimate the upper bound of the fractional derivative operator $\partial_{t}^{\alpha}$. Now we introduce the functions

$$
\begin{aligned}
& \Psi_{n}(t):=\left\|\frac{\partial^{2} \phi_{1 n}}{\partial z^{2}}(t, \cdot)\right\|_{L^{2}}^{2}+\left\|\frac{\partial^{2} \phi_{2 n}}{\partial z^{2}}(t, \cdot)\right\|_{L^{2}}^{2}, \\
& \Phi_{n}(t):=\left\|\frac{\partial \phi_{1 n}}{\partial z}(t, \cdot)\right\|_{L^{2}}^{2}+\left\|\frac{\partial \phi_{2 n}}{\partial z}(t, \cdot)\right\|_{L^{2}}^{2} \cdot
\end{aligned}
$$

Operating on (4.8) by $I^{\alpha}$ and employing the Sobolev inequality yield

$$
\begin{aligned}
\Phi_{n}(t) & \leq \Phi_{0}+\sigma\left(\int_{0}^{t} \frac{(t-\tau)^{\alpha-1}}{\Gamma(\alpha)} \Phi_{n}(\tau) d \tau+\int_{0}^{t} \frac{(t-\tau)^{\alpha-1}}{\Gamma(\alpha)} \Psi_{n}(\tau) d \tau\right) \\
& \leq \Phi_{0}+\frac{\sigma T^{\alpha}}{\Gamma(\alpha+1)} \sup _{t \in[0, T]} \Phi_{n}(t)+\sigma \int_{0}^{t} \frac{(t-\tau)^{\alpha-1}}{\Gamma(\alpha)} \Psi_{n}(\tau) d \tau .
\end{aligned}
$$

A computation implies that

$$
\begin{gathered}
\sup _{t \in[0, T]} \Phi_{n}(t) \leq \frac{\Phi_{0}}{1-\frac{\sigma T^{\alpha}}{\Gamma(\alpha+1)}}+\frac{\sigma}{1-\frac{\sigma T^{\alpha}}{\Gamma(\alpha+1)}} \int_{0}^{t} \frac{(t-\tau)^{\alpha-1}}{\Gamma(\alpha)} \Psi_{n}(\tau) d \tau \\
:=M_{0}+M_{1} \sup _{t \in(0, T]} \int_{0}^{t} \frac{(t-\tau)^{\alpha-1}}{\Gamma(\alpha)} \Psi_{n}(\tau) d \tau .
\end{gathered}
$$


By induction, we find that for all $t \in[0, T]$ and $n$

$$
\sup _{n} \sup _{t \in[0, T]} \Phi_{n}(t) \leq M E_{\alpha, 1}(M T)
$$

where $M$ is a positive constant depending on $\alpha, \sigma, \Phi_{0}$. Thus, we have

$$
\sup _{n}\left(\left\|\partial_{t}^{\alpha} \phi_{1 n}(t, \cdot)\right\|_{L^{2}}+\left\|\partial_{t}^{\alpha} \phi_{2 n}(t, \cdot)\right\|_{L^{2}}\right) \leq M_{T, \alpha} \cdot
$$

\section{Step 5. Convergence of the approximate solution. Let}

$$
\Lambda_{n}:=\left\|\phi_{1 n}(t, \cdot)\right\|_{L^{2}}^{2}+\left\|\phi_{2 n}(t, \cdot)\right\|_{L^{2}}^{2}
$$

the Young inequality shows

$$
\partial_{t}^{\alpha} \Lambda_{n}(t) \leq L\left(\Lambda_{n}(t)+\Lambda_{n-1}(t)\right)
$$

Applying the Gronwall lemma to (4.13), we attain

$$
\Lambda_{n}(t) \leq \widetilde{L}_{\alpha} \int_{0}^{t} \Lambda_{n-1}(s) d s,
$$

where $\widetilde{L}_{\alpha}$ is a constant depending on all the coefficients of the system (3.1a)-(3.1b) and its initial condition. Thus we have

$$
\Lambda_{n}(t) \leq \frac{\left(\widetilde{L}_{\alpha} t\right)^{n-1}}{(n-1) !} \sup _{s \in[0, T]} \Lambda_{1}(s)
$$

therefore, the sequence $\left(\phi_{1 n}, \phi_{2 n}\right)$ is a Cauchy sequence in the space $L^{\infty}\left([0, T], L^{2}(U)\right)^{2}$. This completes the existence proof. The next step shows the uniqueness.

Step 6. Uniqueness. Let $\left(\phi_{1}, \phi_{2}\right)$ and $\left(\varphi_{1}, \varphi_{2}\right)$ be two solutions for the system (3.1a)-(3.1b) with the same initial condition $\left(\phi_{1}^{0}, \phi_{2}^{0}\right) \in H^{1}(U)$. Assume that $u=\phi_{1}-\varphi_{1}$ and $v=\phi_{2}-\varphi_{2}$ satisfy the system

$$
\begin{aligned}
& i \partial_{t}^{\alpha} u=-\frac{1}{2} \Delta u+\frac{\beta^{2}}{2}|z|^{2} u+\gamma_{11}|u|^{\mu} u+\gamma_{12}|v|^{2} u+\lambda v, \\
& i \partial_{t}^{\alpha} v=-\frac{1}{2} \Delta v+\frac{\beta^{2}}{2}|z|^{2} v+\gamma_{12}|u|^{2} v+\gamma_{22}|v|^{\mu} v+\lambda u .
\end{aligned}
$$

As in Step 1, we multiply (4.15a), (4.15b) by $u, v$, respectively, integrate over $U$ and sum up, and we conclude that

$$
\begin{aligned}
\frac{1}{2} \partial_{t}^{\alpha}( & \left.\|u(t, \cdot)\|_{L^{2}}^{2}+\|v(t, \cdot)\|_{L^{2}}^{2}\right) \\
\quad \leq & \frac{1}{2}\left(\|\nabla u(t, \cdot)\|_{L^{2}}^{2}+\|\nabla v(t, \cdot)\|_{L^{2}}^{2}\right)+\left(|\lambda|+\frac{\beta^{2}}{2}\right)\left(\|u(t, \cdot)\|_{L^{2}}+\|v(t, \cdot)\|_{L^{2}}\right) \\
& +\left(\frac{K_{\mu}}{\mu+2}+K\right)\left|\gamma_{12}\right|\left(\|\nabla u(t, \cdot)\|_{L^{2}}^{m_{1}}\|u(t, \cdot)\|_{L^{2}}^{2}+\|\nabla v(t, \cdot)\|_{L^{2}}^{m_{2}}\|v(t, \cdot)\|_{L^{2}}^{2}\right) .
\end{aligned}
$$


By applying the generalized Gronwall lemma, we conclude that

$$
\sup _{t \in[0, T]}\left(\|u(t, \cdot)\|_{L^{2}}^{2}+\|v(t, \cdot)\|_{L^{2}}^{2}\right) \leq \rho,
$$

where $\rho$ is an arbitrary constant depending on $\alpha, \beta, \mu, K_{\mu}, K,\left|\gamma_{12}\right|, T,|\lambda|, \phi_{1}^{0}$, and $\phi_{2}^{0}$. Hence we complete the proof.

\section{Numerical scheme}

The system is solved in a bounded interval $J=[a, b]$, where $a<b$. We utilize a grid with size $h$ and grid point $z_{j}=z_{0}+j h, j=0, \ldots, m$, where $m+1 \in \mathbb{N}$ is an odd number of grid points. Consequently, $h=\frac{b-a}{m}$. The time grid is posed by $t_{n}=n s, n \in \mathbb{N}_{0}$, where $s>0$ is the time step measure. We put $\left(\phi_{k}\right)_{j}^{n}:=\phi_{k}\left(z_{j}, t_{n}\right)$, where $k=1,2, j=0, \ldots, m$, and $n \in \mathbb{N}_{0}$. We split the system (3.1a)-(3.1b) into the three subsystems

$$
\begin{aligned}
& i \partial_{t}^{\alpha} \phi_{1}=\frac{\beta^{2}}{2}|z|^{2} \phi_{1}+\gamma_{11}\left|\phi_{1}\right|^{\mu} \phi_{1}+\gamma_{12}\left|\phi_{2}\right|^{2} \phi_{1}, \\
& i \partial_{t}^{\alpha} \phi_{2}=\frac{\beta^{2}}{2}|z|^{2} \phi_{2}+\gamma_{12}\left|\phi_{1}\right|^{2} \phi_{2}+\gamma_{22}\left|\phi_{2}\right|^{\mu} \phi_{2}, \\
& i \partial_{t}^{\alpha} \phi_{k}=-\frac{1}{2} \triangle \phi_{k}, \quad k=1,2, \\
& i \partial_{t}^{\alpha} \phi_{1}=\lambda \phi_{2}, \quad i \partial_{t}^{\alpha} \phi_{2}=\lambda \phi_{1},
\end{aligned}
$$

assumed on $\left[t_{n}, t_{n+1}\right]$ and subject to some initial conditions. We may solve these systems as follows:

Step 1. Equations (5.1a) and (5.1b) can be solved by using numerical methods (see [16]); thus we get an approximate solution in the interval $\left[t_{n}, t_{n}+\frac{s}{2}\right]$,

$$
\left(\phi_{k}\right)_{j}^{\diamond} \approx E_{\alpha, 1}\left(\frac{-i s^{\alpha}}{2}\left(\frac{\beta^{2}}{2} z_{j}^{2}+\gamma_{11}\left|\left(\phi_{1}\right)_{j}^{n}\right|^{\mu}+\gamma_{12}\left|\left(\phi_{2}\right)_{j}^{n}\right|^{2}\right)\right)\left(\phi_{1}\right)_{j}^{n}
$$

where

$$
E_{\alpha, \rho}(z)=\sum_{k=0}^{\infty} \frac{z^{k}}{\Gamma(\alpha k+\rho)}
$$

is the Mittag-Leffler function. Similarly for $\left(\phi_{2}\right)_{j}^{\diamond}$.

Step 2. Equation (5.2) can be solved, in the interval $\left[t_{n}, t_{n+1}\right]$, by using the method suggested in [17], yielding

$$
\left(\phi_{k}\right)_{j}^{\diamond \diamond}=\sum_{m=1}^{\infty} E_{\alpha, 1}\left(\frac{-i s^{\alpha}}{2}\left(\frac{\pi}{2}\right)^{2} m^{2}\right) \sin \left(\frac{m \pi z}{2}\right) \int_{0}^{1}\left(\phi_{k}\right)_{j}^{\diamond}(t, w) \sin \left(\frac{m \pi w}{2}\right) d w,
$$

for some initial conditions depending on $\left(\phi_{k}\right)_{j}^{\diamond}$.

Step 3. The equations of (5.3) are solved in the interval $\left[t_{n}, t_{n+1}\right]$ implying

$$
\begin{aligned}
& \left(\phi_{1}\right)_{j}^{\infty \infty}=\cos _{\alpha}(\lambda s)\left(\phi_{1}\right)_{j}^{\infty \diamond}+i \sin _{\alpha}(\lambda s)\left(\phi_{2}\right)_{j}^{\infty \diamond,} \\
& \left(\phi_{2}\right)_{j}^{\infty \infty \diamond}=i \sin _{\alpha}(\lambda s)\left(\phi_{1}\right)_{j}^{\infty \diamond}+\cos _{\alpha}(\lambda s)\left(\phi_{2}\right)_{j}^{\infty \diamond},
\end{aligned}
$$

where $\cos _{\alpha}$ and $\sin _{\alpha}$ are the fractional trigonometric functions [3]. 


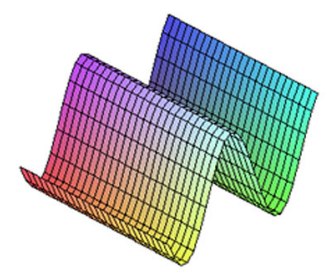

(a)

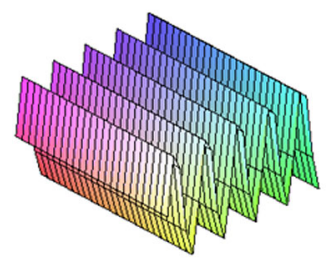

(c)

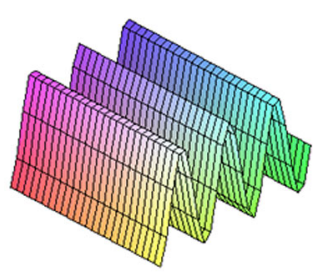

(b)

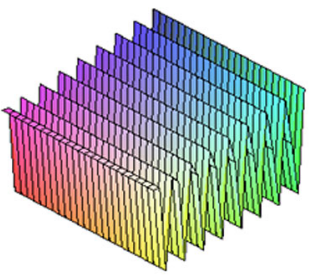

(d)

Figure 1 Numerical solutions of the system (3.1a)-(3.1b). Solutions of the system (3.1a)-(3.1b), for different values of $\alpha \in(0,1)$. They are plotted in three dimensions, $t \in[0,5]$, with the initial wave function of a quantum system $\phi_{1}^{0}=\phi_{2}^{0}=0$ and $z=(x, y), 0 \leq x<1,0 \leq y<1$. The iterations $k$ of the algorithm are taken from 1 to 1,000 . (a) $\alpha=0.1$, (b) $\alpha=0.5$, (c) $\alpha=0.75$, and (d) $\alpha=0.9$. We conclude that the increasing value of $\alpha$ implies increasing wavelets of solutions.

Step 4. We solve the problem (5.1a) and (5.1b) in the interval $\left[t_{n}+\frac{s}{2}, t_{n+1}\right]$ employing $\left(\phi_{1}\right)_{j}^{\infty \diamond \diamond}$ to introduce $\left(\phi_{1}\right)_{j}^{\infty \diamond \diamond}$.

Step 5. Similarly, we solve (5.2) and (5.3) in the interval $\left[t_{n}+\frac{s}{2}, t_{n+1}\right]$ to imply $\left(\phi_{k}\right)_{j}^{n+1}$ (see Figure 1).

\section{Competing interests}

The authors declare that they have no competing interests.

\section{Authors' contributions}

The two authors jointly worked on deriving the results and approved the final manuscript.

\section{Author details}

${ }^{1}$ Institute of Mathematical Sciences, University of Malaya, Kuala Lumpur, 50603, Malaysia. ${ }^{2}$ Faculty of Computer Science and Information Technology, University of Malaya, Kuala Lumpur, 50603, Malaysia.

\section{Acknowledgements}

The authors would like to thank the referees for giving useful suggestions for improving the work. This research is supported by Project No.: RG312-14AFR from the University of Malaya.

Received: 13 July 2014 Accepted: 6 January 2015 Published online: 22 January 2015

\section{References}

1. Metzler, R, Klafter, J: The random walkers guide to anomalous diffusion: a fractional dynamics approach. Phys. Rep. $339,1(2000)$

2. Klafter, J, Shlesinger, MF, Zumofen, G: Beyond Brownian motion. Phys. Today 49, 33 (1996)

3. Kilbas, AA, Srivastava, HM, Trujillo, Jj: Theory and Applications of Fractional Differential Equations. North-Holland Mathematical Studies, vol. 204. Elsevier/North-Holland, Amsterdam (2006)

4. Podlubny, I: Fractional Differential Equations. Academic Press, New York (1999)

5. West, BJ, Bologna, M, Grigolini, P: Physics of Fractal Operators. Springer, New York (2003)

6. Laskin, N: Fractals and quantum mechanics. Chaos 10, 780 (2000)

7. Guo, X, Xu, M: Some physical applications of fractional Schrödinger equation. J. Math. Phys. 47, 082104 (2006)

8. Dong, J, Xu, M: Some solutions to the space fractional Schrödinger equation using momentum representation method. J. Math. Phys. 48, 072105 (2007)

9. Liu, Y, Wang, L: Boundedness for Riesz transform associated with Schrödinger operators and its commutator on weighted Morrey spaces related to certain nonnegative potentials. J. Inequal. Appl. 2014, 194 (2014)

10. Zhao, T: Minimally thin sets associated with the stationary Schrödinger operator. J. Inequal. Appl. 2014, 67 (2014) 
11. Yang, H, Zhu, S: Blow-up criteria for the inhomogeneous nonlinear Schrödinger equation. J. Inequal. Appl. 2014, 55 (2014)

12. Ford, NJ, Rodrigues, MM, Vieira, N: A numerical method for the fractional Schrödinger type equation of spatial dimension two. Fract. Calc. Appl. Anal. 16(2), 454-468 (2013)

13. Wang, D, Xiao, A, Yang, W: Crank-Nicolson difference scheme for the coupled nonlinear Schrödinger equations with the Riesz space fractional derivative. J. Comput. Phys. 242(1), 670-681 (2013)

14. Alsaedi, A, Ahmad, B, Kirane, M: Maximum principle for certain generalized time and space-fractional diffusion equations. Q. Appl. Math. (to appear)

15. Antonelli, P, Weishaäupl, R: Asymptotic behavior of nonlinear Schrödinger system with linear coupling. arXiv:1303.6427v2 [math.AP] (2013)

16. Ibrahim, RW: Numerical solution for complex systems of fractional order. J. Appl. Math. 2012, 678174 (2012)

17. Du, R, Cao, WR, Sun, ZZ: A compact difference scheme for the fractional diffusion-wave equation. Appl. Math. Model. 34, 2998-3007 (2010)

Submit your manuscript to a SpringerOpen ${ }^{\circ}$ journal and benefit from:

- Convenient online submission

Rigorous peer review

- Immediate publication on acceptance

- Open access: articles freely available online

- High visibility within the field

- Retaining the copyright to your article 